\title{
The Construction of Autonomous Virtual Hand in Virtual Assembly Environments
}

\author{
Zhang Ting, Cheng Cheng, Ge Wei, and Zhang Jing
}

\begin{abstract}
This paper introduces a method of constructing autonomous virtual hand in virtual assembly environments. The goal of this research is presented to improve the practicability of virtual hand in virtual environments. The autonomous virtual hand is realized as the agent by three steps. A gesture set of virtual hand is constructed. Initial gesture, basic gesture and grasping gestures help virtual hand to achieve autonomous grasping. Perceptional ring is constructed for virtual hand, which support the autonomous virtual hand sense the virtual object. The system divides autonomous grasping process into three perception stages. By using this method, user heavy load will be greatly reduced in the assembly process. We prove that this autonomous grasping behavior of flexible virtual hand has a good availability and efficiency.
\end{abstract}

Index Terms-Human-computer-interaction, virtual environment, virtual assembly, agent, autonomous virtual hand, behavior, perception.

\section{INTRODUCTION}

To realize natural and harmonious human-computer interaction in virtual assembly, an autonomous virtual hand system has been developed. 3D mouse is used to manipulate the virtual hand parts. 3D mouse consist of control cap and keys, in which different key corresponds to different function. Users can manipulate control cap to perform continuous operation to virtual hand.

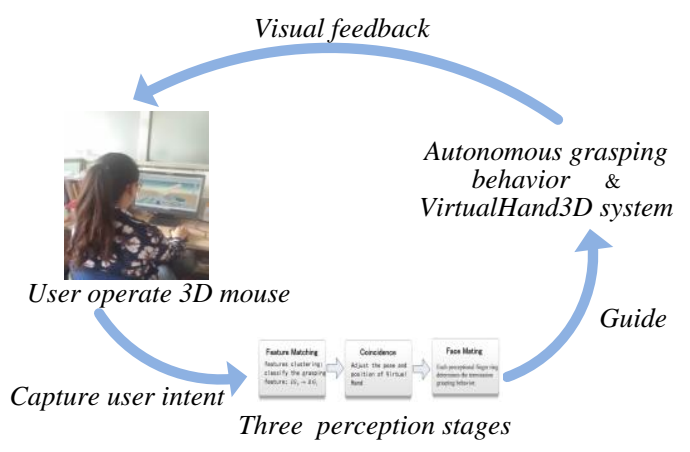

Fig. 1. VirtualHand3D system.

The paradigm of system VirtualHand3D manipulation is shown in Fig. 1. In our VirtualHand3D system, users could control 3D mouse to drive the virtual hand to be closed to a

Manuscript received August 9, 2016; revised December 9, 2016. This work was financially supported by National Science Foundation under China No. 61370135. Besides, this paper is also partially supported by Beijing Key Discipline Program.

All authors are with the School of Computer Science, Beijing Laboratory of Intelligent Information of Technology, Beijing Institute of Technology, China (e-mail: zhang_ting0402@163.com, guoguocheng@vip.sina.com, gewei_hello@163.com, cailingjingjing@163.com). part. If the distance between the virtual hand and the target part is less than a threshold, the system will automatically light the target part's surrounding box. Then, users utilize the 3D mouse to release a grasping command. Virtual hand senses features of the part. Three stage perceptions, Feature Matching, Coincidence and Face Mating, achieve an autonomous grasping behavior.

\section{RELATED WORK}

Virtual hand, as an important metaphor, plays an indispensable role and brings us immersion and realism in environments. In virtual assembly system, the popular studies are mainly that users utilizes data glove [1], [2] to control virtual hand or image processing technigue [3], [4] to track bare hand movement. However, this kind of animation is not suitable for daily interaction in engineering, and has some defect, such as user felt uncomfortable; the simulation of hand is not flexible and it also has bad real-time performance. In addition, current virtual hand causes a heavy user operation load. So, this paper will study the software construction of autonomous virtual hand and elements state that the structure of virtual hand is a practical strategy.

Designing virtual hand involves two major research directions: gestures [5] and collision detection [6], [7] between virtual hand and part. In the graphic filed, Borst and Indugula [8] approach couples tracked hand configuration to a simulation-controlled articulated hand model. With respect to the gestures of virtual hand, Beifang Yi, etc. [9] made a research and summary about the real-time gestures design. Besides, the paper also states how with bone and muscle. Collision detection is one of the key to ensure correct interaction in virtual environments. It usually uses the bounding box to sense the surrounding environment [10]. But, this method has a bad real-time response in most situations. Cheng Cheng [11] pointed out that the virtual object should have the independent ability to sense surrounding objects. Kanav Kahol [12] proposes that using sensitivity cue to convey the part's effective information, such as size, shape, texture and material. Fons Kuijk and Konstantions C [13] constructed the agents in a virtual environment and make them like human being perceive and communication. Inspired by agent, we make our virtual hand become the agent and which have perceive and grasping in assembly system.

\section{AUTONOMOUS VIRTUAL HAND}

The process virtual hand grasping part is a continuous operation, can be extracted into many different gestures. In our system, we define three types of gestures: initial gesture, 
basic gesture and final gesture. In addition, it is through three types gesture conversions to realize virtual hand whole autonomous grasping behavior.

\section{A. Constructing the Gestures Set of Virtual Hand}

Based on industrial products' geometric features and the grasping experience in our daily life, we construct the virtual hand gesture set. According to the statistics and analysis of grasping gestures in our daily life, Cheng [14] defines a frequently-used virtual hand gestures set in Fig. 2: GeSet= \{Pnt, 2fNip, 3fNip, 4fNip, 5fNip, AGrab, PGrab, Grasp \}. Pnt is initial gesture from which every grasping gesture can be derived. GeSet is virtual hand's basic gesture. The difference between final grasping gesture and basic gesture is the fingers' grasping depth and angle.

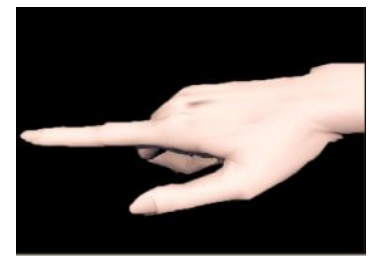

(a) Pnt

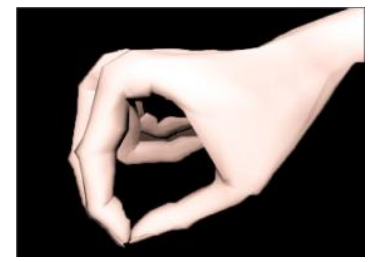

(c) $3 \mathrm{fNip}$

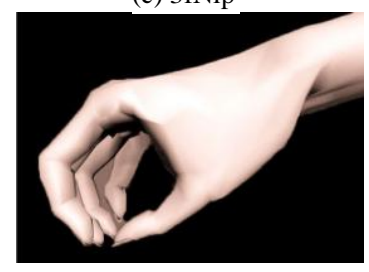

(e) $5 \mathrm{fNip}$

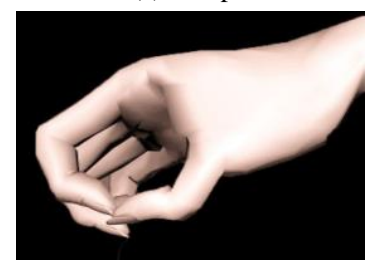

(g) PGrab

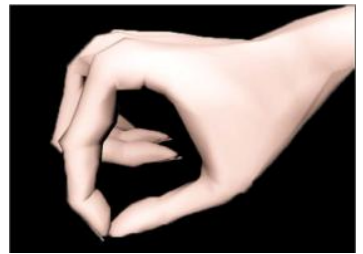

(b) $2 \mathrm{fNip}$

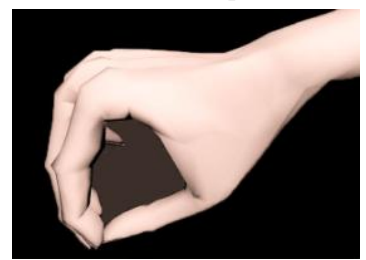

(d) 4 fNip

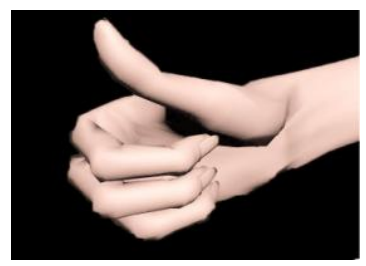

(f) AGrab

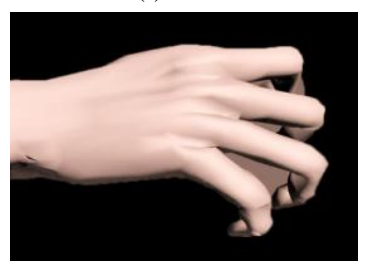

(h) Grasp
Fig. 2. The gestures set of virtual hand.

TABLE I: FEATURE TO GESTURES MAPPING

\begin{tabular}{lll}
\hline \hline Feature & Concave/Convex & Gestures \\
\hline Cylinder & Convex & AGrab \\
Block & Convex & PGrab \\
Pipe & Convex & AGrab \\
Shaft & Convex & PGrab \\
Prism & Convex & $2 \mathrm{fNip}$ \\
Gear & Convex & $3 \mathrm{fNip}$ \\
Ball & Convex & Grasp \\
SquareWasher & Concave & $3 \mathrm{fNip}$ \\
RoundWasher & Concave & $3 \mathrm{fNip}$ \\
Hole & Concave & $2 \mathrm{fNip}$ \\
Disc & Convex & PGrab \\
\hline \hline
\end{tabular}

Virtual hand acquires feature information from target part, then change from the initial gesture to the basic gestures in

grasping process. Mapping table between the basic gestures and feature should be built. Through repeated experiments in VirtualHand3D system, it is discovered that the concavity, shape, size and weight of feature mainly affect the basic gesture of virtual hand. The rules see for mapping features to a certain basic gesture: see in Table I. For simplicity reason, the authors only show the major geometric property in Table I.

\section{B. Constructing the Perceptional Ring for Virtual Hand in} Face Mating Perception Stages

The process that virtual hand converts from basic gestures into final gestures involves a perceptional procedure. Perceptional ring are built for every finger. The virtual rings designed is shown by Fig. 3(a), and the usage of finger rings in dynamic calculation of face mating perception is shown with Fig. 3(b).

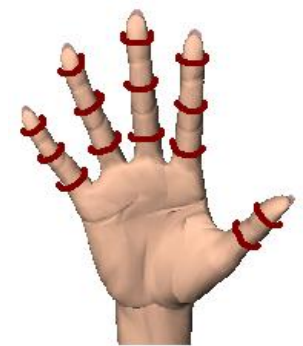

(a)

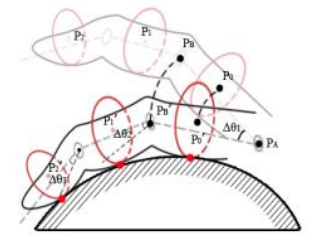

(b)

Fig. 3. Perceptional ring and perceptional point.

In actually assembly process, each time the interaction between virtual hand and the part will undergo this three stages perception: a) Feature Matching Perception is that virtual hand according to user intent identifies the set feature and extract feature parameters. According to the feature gestures mapping rules, virtual hand will change from the initial gesture $\left(I G_{0}\right)$ into basic gestures $\left(B G_{i}\right)$; b) Coincidence perception is that virtual hand adjust its orientation. To prepare for implementation of grasping movement, virtual hand will automatically move to the target part and adjust to suitable position and orientation; c) Face Mating Perception, is that, virtual hand control and drive fingers move. And every individual finger ring is used to determine the final position of every finger segment $\left(F G C_{i}\right)$. Virtual hand has a better perceptional mechanism which is consisted of these three stage perceptions. The framework of this three stage perceptions is given in Fig. 4.

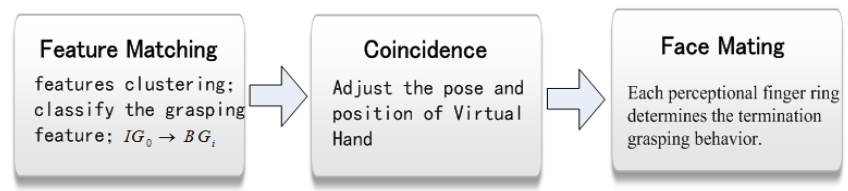

Fig. 4. Three perception stages in virtual hand.

\section{Autonomous Grasping Algorithm}

Autonomous virtual hand will be an important development in virtual assembly system and play an irreplaceable role in virtual world. Autonomous virtual hand could sense the feature of part and according to this information, virtual hand could select the correct grasping gesture. The algorithm is described as below in Fig. 5 . 


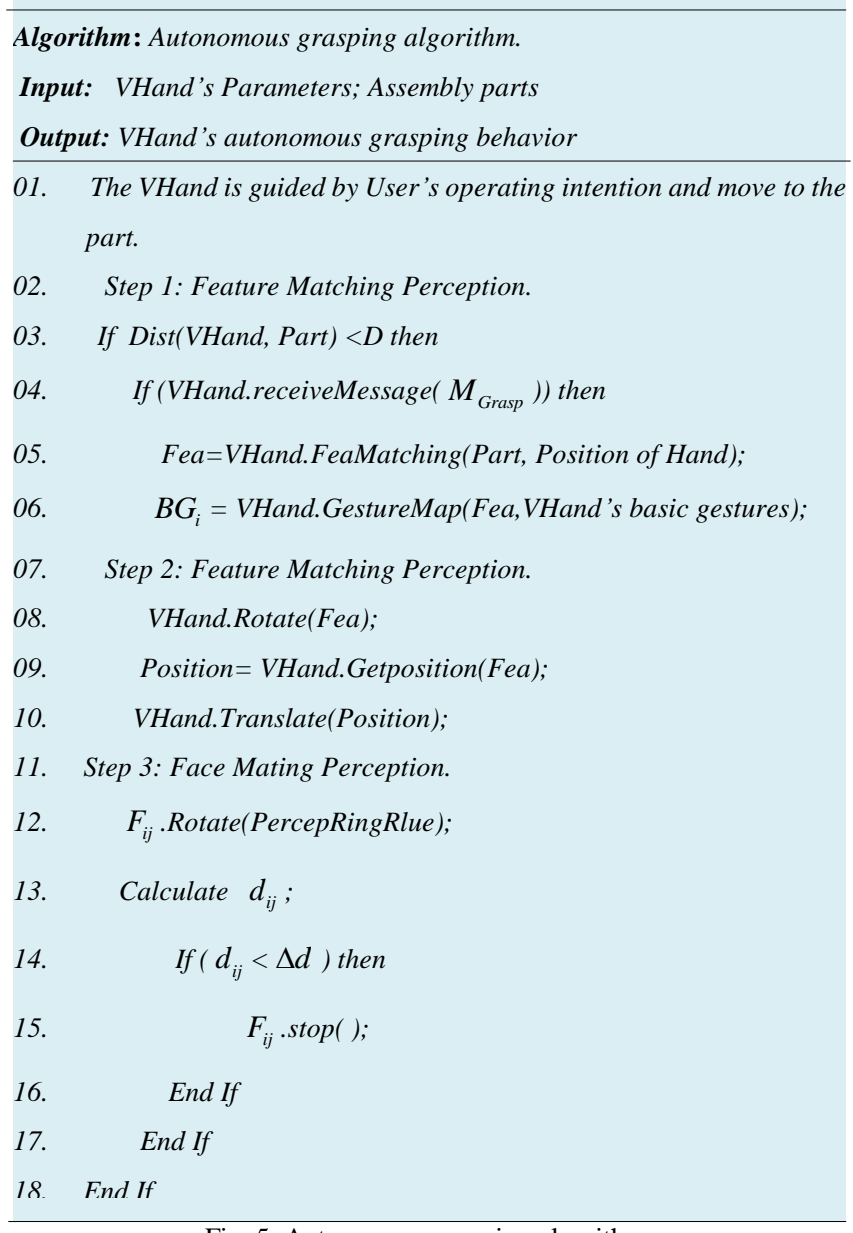

Fig. 5. Autonomous grasping algorithm. (a) Initial gesture

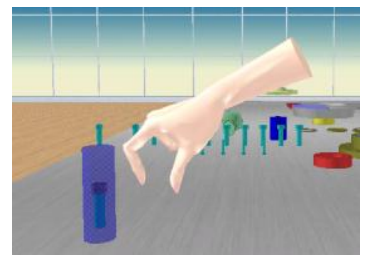

(c) Initial gesture to basic gesture

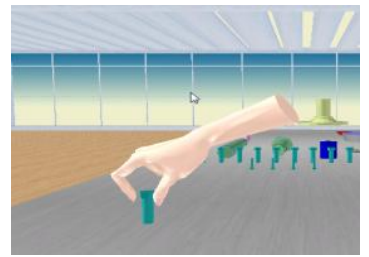

(e) Grasping

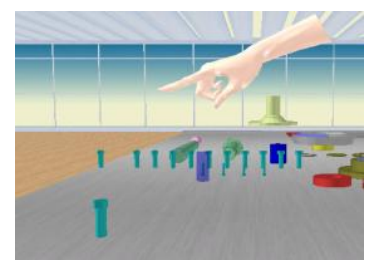

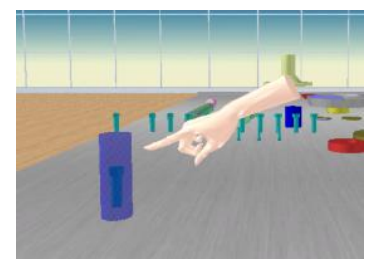

(b) Recognition feature

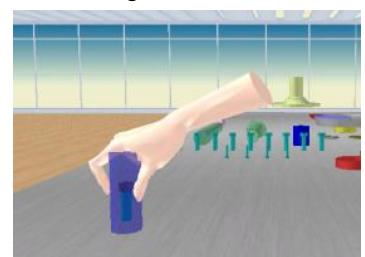

(d) Adjust position and orientation

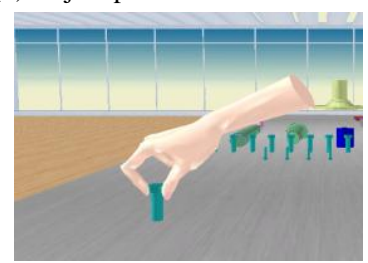

(f) Completed Grasping
Fig. 6. Process of autonomous grasping a bolt.

$I G_{0}$ represents the initial gesture, $B G_{i}$ represents basic gestures, $F G C_{i}$ represents the final gestures; the function of Dist ( ) calculates the distance between virtual hand and the target part; $M_{\text {Grasp }}$ represents the grasping command that the user use released use $3 \mathrm{D}$ mouse; D is a threshold. In this grasping process, each finger performs individual Face
Mating perception, $F_{i j}$ represents a finger segment number $i$ $(i=1,2, \ldots, 5$ represents finger $)$ number $j, j=1,2,3$ represents the segments ). The function $F_{i j}$.stop ( ) says that all knuckles have completed the Face Mating Perception.

The following pictures in Fig. 6 show the process that virtual hand autonomously grasp the target part: Fig. 6a) The initial gesture of virtual hand $\left(I G_{0}\right)$ is pointing gesture, the distance between virtual hand and the part is greater than $\mathrm{D}$; Fig. 6b) Virtual hand move to a target part and automatically sense the feature on the target part. The part's bounding box is highlighted when the Dist (VHand, Part) is less than D; Fig. 6c) virtual hand determines the grasping feature. Virtual hand changes from the initial gesture to a basic gesture. Fig. 6d) Virtual hand automatically moves to the target part and adjust position and orientation. Fig. 6e) Virtual hand start to grasp the part. Each individual perception finger ring in virtual hand determines the real-time eventually grasping behavior; Fig. 6f) The final grasping gesture.

\section{EXPERIMENTS AND ANALYSIS}

In VirtualHand3D system, 145 assembly parts which contains 15 types of features are involved. Autonomous virtual hand we designed evolve from original gesture to basic gestures and from basic gestures to final gestures, and be able to perfectly grasp these 145 parts. Fig. 7 demonstrates autonomous graspings of some types of parts and features in the system.
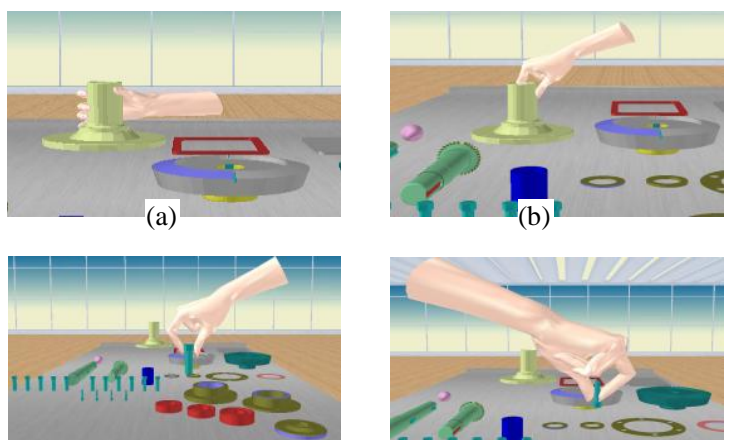

(c)

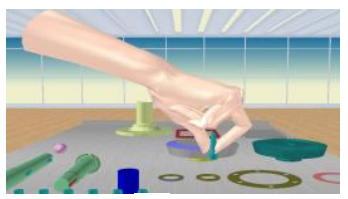

(d)

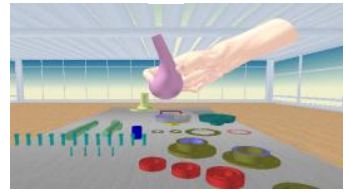

(e)
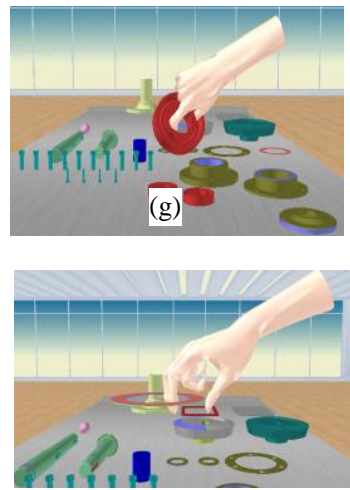

(i)

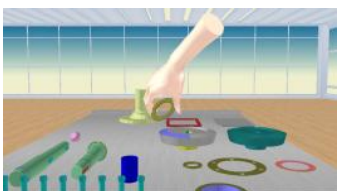

(f)
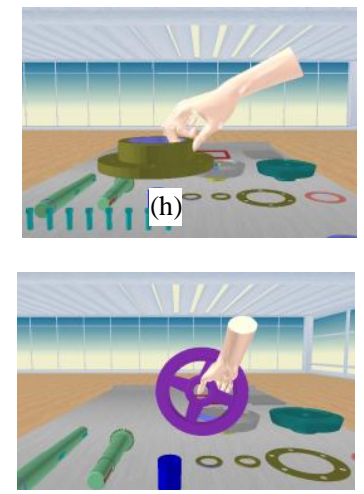

(j)

Fig. 7. Demos of features autonomous grasping. 
A set of metrics have been established to evaluate the capabilities of the virtual hand: see Table II. It is compared with collision detection based virtual hands. These data are obtained through grasping different parts proves that this autonomous grasping behavior of a flexible virtual hand is available and efficient.

TABLE II: COMPARE VIRTUALHAND3D WITH TRADITIONAL VIRTUAL HANDS

\begin{tabular}{|c|c|c|c|c|c|}
\hline Object & Input Device & Reliability & Op-load & Real-time Frame/s & Seman-tics \\
\hline $\begin{array}{c}\text { Tradition } \\
\text { Virtual hand }\end{array}$ & Data glove & General & 13.5 & 1 & 1 \\
\hline $\begin{array}{l}\text { Auto- } \\
\text { 3Dhand }\end{array}$ & 3D mouse & Perfect & 3 & 70 & 5.4 \\
\hline
\end{tabular}

1) Reliability metric: The popular way is that the user wear data glove to control virtual hand to grasp a part in assembly environments. However, this kind of animations has a time-delay, and the transformation of gestures was reflected too slowly. The relia-bility of traditional virtual hand is general. In our VirtualHand3D system, autonomous grasping behavior of flexible virtual hand is available and efficient through grasping different parts. Our system constructs the gestures set of virtual hand and could quickly transform between these gestures by making use of Features to Gestures mapping. Therefore, the relia-bility of VirtualHand3D is perfect.

2) Op-load metric: During the grasping process in virtual assembly environments, the traditional method makes use of data glove. The number of sensors of data glove determines the op-load metric. If every knuckle has a sensor, there are 14 sensors in data glove and the feedback events depend on the numbers of sensors. In traditional system, the statistical average Op-load time is 13.5. VirtualHand3D runs on the device of which the operation system is Windows 7 32-bit, the RAM of which is $4.00 \mathrm{~GB}$ and the processor of which is the $2.93 \mathrm{G} \mathrm{Hz}$ of Intel CPU. VirtualHand3D captures the grasping intent and get feedback from Feature Matching perception, Coincidence perception and Face Mating Perception. The grasping process produces 3 visual feedback events and the op-load metric is 3.0 in VirtualHand3D system.

3) Real-time metric: Traditional virtual hand use collision detection to detect the surrounding environments and the real-time of which is the frame/s of 1 . Obviously, traditional virtual hand is not suitable for interactions in daily engineering design activities. But, the VirtualHand3D used perceptional ring to sense the surrounding environments and frame/s is 70 . Clearly, it is real-time.

4) Semantics metric: the feedbacks of tradition virtual hand mechanisms are only contacts points, the value of seman-tics is 1 . On the other hand, the VirtualHand3D can sense the distribution and types of feature, the geometry information of the part, the grasping gestures and position, etc. So the statistical average value of seman-tics is 5.4. It could perfectly recognize the surrounding environments.

To sum up, the VirtualHand3D is low cost, has small operation load, high perception is efficiency. It has a better flexible and interactive capacity. Besides, the VirtualHand3D could adapt to the needs of grasping operation in assembly system.

\section{CONCLUSION}

This paper has proposed a new perception mechanism and a new strategy of autonomous virtual hand behavior. The virtual hand in VirtualHand3D system can be used in any virtual environments and only need simple input devices like 3D space mouse. The VirtualHand3D autonomous virtual hand has perception mechanism, action mechanism and constraint mechanism. Also, the virtual hand could be able to capture the user's intent and has real-time interactive performance. We will further investigate the behaviors of the virtual hand and try to simulate all the detail actions of virtual hand to make it more possible to validate the assembly.

\section{ACKNOWLEDGMENT}

Zhang Ting Author thanks all those who helped her during the writing of this thesis. Zhang Ting Author gratefully acknowledges the help of her postgraduate tutor teacher, Mr. Cheng Cheng, Who has offered her valuable suggestions in the academic studies. In the preparation of this paper, he has spent much time reading through each draft and provided me with inspiring advice. Without his patient instruction, insightful criticism and expert guidance, the completion of this paper would not have been possible.

Zhang Ting Author should also thank her postgraduate classmates, Ge Wei and Zhang Jing, who have instructed and help her a lot in the past one year.

\section{REFERENCES}

[1] C. S. Fahn and H. Sun, "Development of a data glove with reducing sensors based on magnetic induction," IEEE Trans. on Industrial Electronics., vol. 52, no. 2, pp. 589-594, April 2005.

[2] Y. Liu and G. Wan, "Techniques for selecting and manipulating object in virtual environment based on 3-DOF trackers and data glove," in Proc. 16th International Conference on Artificial Reality and Telexistence-Workshops (ICA'06), 2006, pp. 662-665.

[3] A. Cassidy, D. Hook, A. Baliga, and T. Chen, "Hand tracking using spatial gesture modeling and visual feedback for a virtual DJ system," in Proc. IEEE International Conference on Multimodal Interfaces, 2002, pp. 197-197

[4] T. Heap and D. Hogg, "Towards 3D hand tracking using a deformable model," in Proc. the Second International Conference on Automatic Face and Gesture Recognition,1996, pp. 140-145.

[5] M. Weber, G. Heumer, H. B. Amor, and B. Jung, "An animation system for imitation of object grasping in virtual reality," in Proc. 16th International Conference on Artificial Reality and Telexistence, 2006 pp. 65-76.

[6] S. Kockara, T. Halic, K. Iqbal, C. Bayrak, and R. Rowe, "Collision detection: A survey," in Proc. 2007 IEEE International Conference on System, 2007, pp. 4046-4051.

[7] J. Li, B. Gao, and S. Guo, "Design of collision detection algorithms and force feedback for a virtual reality training intervention operation system," in Proc. 2015 IEEE International Conference on Robotics and Biomimetics (ROBIO), 2015, pp. 1660-1665.

[8] C. W. Borst and A. P. Indugula, "Realistic virtual grasping," in IEEE Proc. Virtual Reality, 2005, pp. 91-98. 
[9] B. F. Yi, F. C. Harris, L. Wang, and Y. S. Yan, "Real-time natural hand gestures," Computing in Science \& Engineering, vol. 7, no. 3, pp. 92-96, May-June 2005.

[10] M. Matthew and W. Jane, "Collision detection and response for computer animation," in Proc. 15th Annual conference on Computer Graphics and Interactive Techniques, 1998, pp. 289-298.

[11] C. Cheng, R. Jiang, and X. M. Dong, "Human knowledge acquisition from 3D interaction in virtual environments," Science China: Information Sciences., vol. 55, no. 7, pp. 1528-1540, July 2012.

[12] M. Fujita and O. Koike, "Computation of Capillary Interactions among Particles at Free Surface," Japan Society of Applied Physics through the Institute of Pure and Applied Physics, vol. 6, no. 3, pp. 03651(4.pp), March 2013.

[13] F. Kuijk, K. C. Apostolakis, P. Daras, B. Ravenet, H. Wei, and D. S. Monaghan, "Autonomous agents and avatars in REVERIE's virtual environment," in Proc. 20th International Conference on $3 D$ Web Technology, 2015, pp. 179-287.

[14] X. J. Cai and C. Cheng, "Research on behavior simulation of virtual hand in virtual manufacturing environment," Compurer_Aided Design \& Computer Graphics, vol. 27, no. 3, pp. 499-507, March 2015 (in Chinese).

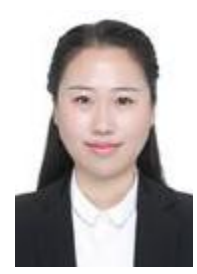

Zhang Ting was born in Heze city of Shandong province in China and also born in 1990. Besides, she is a master candidate in Beijing Laboratory of Intelligent Information of Technology, School of Computer Science, Beijing Institute of Technology. Her degree system is 2.5 year in Beijing, China. Also, her main research topic is the simulation of human-computer interaction and virtual environments. Now, she does research on the construction and simulation behavior of virtual hand and try to simulate all the detail actions of virtual hand like human hand to make it more possible to validate the assembly feasibility.

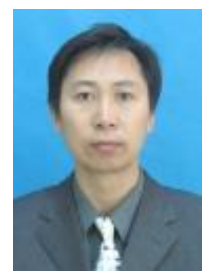

Cheng Cheng was born in Qingdao city of Shandong province in china and also born in 1966. He was a doctor graduated in the School of Computer Science, Chinese Sonica in China. During his working for Ph.D., he was major in the simulation of human-computer interaction and virtual environments. Also, he was an associate professor in Beijing Laboratory of Intelligent Information of Technology,
School of Computer Science, Beijing Institute of Technology in china. Besides, His main research topic is the simulation of human-computer interaction and virtual environments.

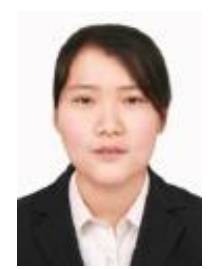

Ge Wei was born in Jining city of Shandong province in China and also born in the year of 1991. Besides, she is a master candidate in Beijing Laboratory of Intelligent Information of Technology, School of Computer Science, Beijing Institute of Technology. Her degree system is 2.5 year in the city of Beijing, China. Also, her main research topic is the simulation of human-computer interaction and virtual environments. Now, she does research on Intent-driven system and experiment evaluation. Sensing the intention of operator could make our system have the ability to sense the surrounding environments and also make our system intelligent.

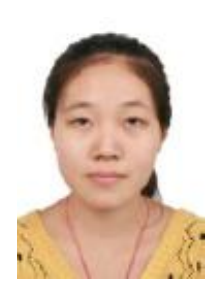

Zhang Jing was born in Heze city of Shandong province in China and also born in the year of 1992. Besides, she is a master candidate in Beijing Laboratory of Intelligent Information of Technology, School of Computer Science, Beijing Institute of Technology. Her degree system is 2.5 year in the city of Beijing, China. Also, her main research topic is the simulation of human-computer interaction and virtual environments. Now, she does research on the virtual assembly. In order to find the problem in the assembly system, operator can verify the component in virtual assembly system and modify the model by using the visual display of the assembly process. 